\title{
Comparison of native myocardial T1 and T2 mapping at 1.5T and $3 T$ in healthy volunteers
}

\section{Reference values and clinical implications}

\author{
Marcel Granitz (D) - Lukas J. Motloch • Christina Granitz · Matthias Meissnitzer · Wolfgang Hitzl • Klaus Hergan · \\ Alexander Schlattau
}

Received: 13 June 2018 / Accepted: 29 October 2018 / Published online: 5 December 2018

(c) The Author(s) 2018

\begin{abstract}
Summary
Background Myocardial native $\mathrm{T} 1$ and $\mathrm{T} 2$ mapping are promising techniques for quantitative assessment of diffuse myocardial pathologies; however, due to conflicting data regarding normal values, routine clinical implementation of this method is still challenging.

Methods To evaluate this situation during daily clinical practice the characteristics of normal values obtained in 60 healthy volunteers who underwent magnetic resonance imaging (MRI) scans on 1.5T and 3T scanners were studied. The T1 modified look-locker inversion recovery (MOLLI; 5(3)3; modified for higher heart rates) and T2 navigator gated black-blood prepared gradient-spin-echo (GraSE) sequences were used.

Results While age and body mass index did not affect relaxation times, a gender and heart rate dependency was found showing higher $\mathrm{T} 1$ and $\mathrm{T} 2$ values in females, whereas at higher heart rates a prolongation of $\mathrm{T} 1$ and a shortening of $\mathrm{T} 2$ relaxation times was found. Particularly prone to artifacts were T2 measurements at $3 \mathrm{~T}$ and the inferolateral wall. In the individual setting mean relaxation times for T1 were $995.8 \pm 30.9 \mathrm{~ms}$ at $1.5 \mathrm{~T}$ and $1183.8 \pm 37.5 \mathrm{~ms}$ at $3 \mathrm{~T}$ and $55.8 \pm 2.8 \mathrm{~ms}$ at $1.5 \mathrm{~T}$ and $51.6 \pm 3 \mathrm{~ms}$ at $3 \mathrm{~T}$ for $\mathrm{T} 2$ indicating a high de-
\end{abstract}

M. Granitz and A. Schlattau contributed equally to this work.

M. Granitz (两) · M. Meissnitzer · K. Hergan · A. Schlattau Department of Radiology, Paracelsus Medical University, Müllner Hauptstraße 48, 5020 Salzburg, Austria m.granitz@salk.at

L. J. Motloch · C. Granitz

Department of Internal Medicine II, Paracelsus Medical University, Salzburg, Austria

\section{W. Hitzl}

Research Office (biostatistics), Paracelsus Medical University, Salzburg, Austria pendency of reference values on MRI protocol when compared to the literature. Furthermore, as presumed mean $\mathrm{T} 1$ and $\mathrm{T} 2$ values correlated in the same individual.

Conclusions The $\mathrm{T} 1$ and $\mathrm{T} 2$ relaxation times depend on physiological factors and especially on MRI protocols. Therefore, reference values should be validated individually in every radiological institution before implementing mapping protocols in daily clinical practice. Correlation of mean $\mathrm{T} 1$ and $\mathrm{T} 2$ values in the same proband at both field strengths indicates intraindividual reproducibility.

Keywords Cardiac $\cdot$ MRI $\cdot$ Normal $\cdot$ Field strength

\section{Introduction}

Cardiac magnetic resonance imaging (CMRI) is the gold standard in noninvasive myocardial tissue characterization [1]; however, early, subtle or diffuse myocardial tissue changes may not be evident using late gadolinium enhancement or conventional scanning techniques which depend on relative signal intensity differences between affected and adjacent, unaffected myocardial tissue [2]. Meanwhile, the use of relaxometry methods has gained widespread acceptance [2-4]. Mapping techniques overcome the dependency on visually distinguishing differences in signal intensities. They allow direct measurement of T1 spin-lattice relaxation time or $\mathrm{T} 2$ spin-spin relaxation time in milliseconds and quantify the signal of each voxel on a standardized scale. Relaxation times depend on the water content in the tissue and allow further tissue characterization. Relaxation times vary in different tissues but also within the same tissue undergoing pathophysiological changes (e.g. inflammation, ischemia, edema and fibrosis) [2]. In addition, unenhanced T1 measurements enable the detection of 
Table 1 Study population characteristics, values are mean \pm SD

\begin{tabular}{|c|c|c|c|}
\hline & Female & Male & $\mathrm{p}$ \\
\hline Total study cohort & 33 & 27 & - \\
\hline Healthy volunteers ${ }^{\mathrm{a}}$ & 32 & 26 & - \\
\hline Age (years) & $40.1 \pm 13.7$ & $42.3 \pm 12.5$ & 0.632 \\
\hline $\mathrm{BMI}\left(\mathrm{kg} / \mathrm{m}^{2}\right)$ & $24.1 \pm 4.3$ & $23.9 \pm 3.7$ & 0.846 \\
\hline Heart rate (bpm) & $65.4 \pm 10.0$ & $62.5 \pm 13.0$ & 0.352 \\
\hline LV end-diastolic volume (ml) & $123.0 \pm 23.2$ & $148.2 \pm 30.1$ & $<0.001$ \\
\hline LV end-systolic volume (ml) & $43.2 \pm 13.6$ & $60.5 \pm 20.7$ & $<0.001$ \\
\hline LV ejection fraction (\%) & $65.6 \pm 5.8$ & $60.0 \pm 6.6$ & 0.001 \\
\hline LV mass (g) & $104.8 \pm 16.0$ & $139.1 \pm 26.2$ & $<0.001$ \\
\hline LV mass index $\left(\mathrm{g} / \mathrm{m}^{2}\right)$ & $58.6 \pm 5.9$ & $72.5 \pm 9.6$ & $<0.001$ \\
\hline RV end-diastolic volume (ml) & $122.9 \pm 26.1$ & $155.2 \pm 28.2$ & $<0.001$ \\
\hline RV end-systolic volume (ml) & $45.4 \pm 13.7$ & $68.4 \pm 18.9$ & $<0.001$ \\
\hline RV ejection fraction (\%) & $63.5 \pm 6.2$ & $57.4 \pm 6.1$ & $<0.001$ \\
\hline RV mass $(\mathrm{g})$ & $52.8 \pm 11.0$ & $71.2 \pm 15.0$ & $<0.001$ \\
\hline $\mathrm{RV}$ mass index $\left(\mathrm{g} / \mathrm{m}^{2}\right)$ & $29.4 \pm 4.6$ & $37.1 \pm 6.4$ & $<0.001$ \\
\hline Interventricular septal thickness (mm) & $8.4 \pm 1.3$ & $9.8 \pm 1.4$ & $<0.001$ \\
\hline
\end{tabular}

various T1-altering substances, such as lipids in Anderson-Fabry disease, proteins in cardiac amyloidosis or iron deposits in hemochromatosis [5-7]. Furthermore, it was shown that native T1-mapping measurements are significantly predictive of all-cause mortality and heart failure events in nonischemic cardiomyopathy [8].

The T2-weighted (T2w) CMRI and T2-weighted short tau inversion recovery (STIR) techniques are well-established sequences for the detection of myocardial edema [9] to differentiate acute from chronic myocardial changes $[10,11]$. The quantitative approach of T2-mapping is a robust, accurate and fast alternative to conventional T2w and STIR sequences for the detection of edema in acute inflammatory cardiomyopathies. This method has the potential to even monitor myocardial inflammation in healing myocarditis [12], which has important clinical implications. Furthermore, it plays a role in the diagnosis of myocardial infarction by overcoming some restrictions of conventional $\mathrm{T} 2 \mathrm{w}$ imaging [4], such as sensitivity to myocardial motion, surface coil intensity variation, high subendocardial signal from static blood, incomplete blood suppression and interreader or intra-reader variability [13].

An important aspect of mapping techniques is that the obtained information has prognostic relevance. The pathology cannot only be detected but also be quantified and the course of disease can be monitored under treatment. Therefore, mapping techniques have the potential to act as biomarkers to facilitate diagnostic decision making (e.g. iron overload, Anderson-Fabry disease) [14].

Despite promising results in the literature, routine implementation of this technique remains challenging. This might be associated with conflicting data regarding normal values. Recent publications suggest that even in sophisticated facilities, a variety of technical, image acquisition and patient-dependent factors influence $\mathrm{T} 1$ and $\mathrm{T} 2$ relaxation times [15]. To evaluate this issue the implementation of relaxometry methods in a general radiology department performing approximately $500 \mathrm{CMRI}$ annually was investigated. To obtain reliable standard values for both $1.5 \mathrm{~T}$ and 3T, the $\mathrm{T} 1$ and $\mathrm{T} 2$ relaxation times were measured in the same cohort using established state of the art mapping sequences.

\section{Subjects and methods}

\section{Study population}

The study protocol was approved by the local ethics committee of the province of Salzburg (Number 415EP/73/412-2014). The cohort consisted of 60 healthy volunteers (33 women/27 men), stratified into two equally sized age groups $<45$ and $\geq 45$ years of age (Table 1). Written informed consent was obtained from all subjects in this study.

Inclusion criteria were no medical history concerning cardiac events, no regular medication, absence of symptoms indicative of cardiovascular conditions and sinus rhythm during the MRI examination. Furthermore, normal cardiac chamber dimensions, normal left ventricular (LV) and right ventricular (RV) wall motion, normal LV and RV ejection fraction as well as normal LV and RV mass were confirmed by the evaluation of CMRI-cine sequences.

Exclusion criteria were general contraindications for MRI (pacemakers, cochlear implants, claustrophobia), pregnancy, consumption of alcohol prior to the examination, known systemic disease and hardness of hearing. 


\section{CMRI protocol}

The CMRI procedure was performed using both a commercially available $1.5 \mathrm{~T}$ and $3 \mathrm{~T}$ scanner $(1.5 \mathrm{~T}$ Ingenia, 3T Achieva, both Philips Healthcare, Best, Netherlands). All volunteers were consecutively examined on two MRI scanners (1.5T and 3T) in a supine position. A combined 16-channel anterior/posterior coil system was used for the $1.5 \mathrm{~T}$ scanner, a 6-channel cardiac RF coil and MultiTransmit technology was used for the 3T scanner. At 3T, in addition to B0 shimming, after B1 calibration RF shimming was applied for better field uniformity. Electrocardiography (ECG) was used for cardiac gating.

All studies were performed by experienced radiographers who were trained in the study protocol.

\section{Cine imaging}

Balanced steady-state free-precession (bSSFP) cine images were obtained in the 4-chamber view and in a stack of short axes (SAX) covering the ventricles. Wall motion was assessed and the LV ejection fraction was quantified. Imaging parameters for $1.5 \mathrm{~T}$ were: repetition time $(\mathrm{TR})=2.8 \mathrm{~ms}$, echo time $(\mathrm{TE})=1.38 \mathrm{~ms}$, flip angle $(\mathrm{FA})=60^{\circ}$, field of view $(\mathrm{FOV})=350 \times 286 \mathrm{~mm}^{2}$, matrix $=176 \times 133$, slice thickness $=8 \mathrm{~mm}$, SENSE factor $=2$ and cardiac phases $=30$. Imaging parameters for 3T were: $\mathrm{TR}=2.8 \mathrm{~ms}, \mathrm{TE}=1.4 \mathrm{~ms}, \mathrm{FA}=45^{\circ}$, $\mathrm{FOV}=320 \times 350 \mathrm{~mm}^{2}$, matrix $=176 \times 208$, slice thickness $=8 \mathrm{~mm}$, SENSE factor $=2$ and cardiac phases $=24$.

\section{T1 mapping}

Data for $\mathrm{T} 1$ mapping were acquired in three SAX slices (apical, mid-ventricular and basal). The balanced SSFP-based modified look-locker inversion recovery (MOLLI) technique was used [16]. Imaging parameters for $1.5 \mathrm{~T}$ were: $\mathrm{TR}=2.2 \mathrm{~ms}, \mathrm{TE}=1.02 \mathrm{~ms}$, $\mathrm{FA}=35^{\circ}, \mathrm{FOV}=380 \times 256 \mathrm{~mm}^{2}$, voxel size $2 \times 2 \times 10 \mathrm{~mm}^{3}$ and SENSE factor $=2$. The MOLLI schema used was 5 beats (3s) 3 beats. Imaging parameters for 3T were: $\mathrm{TR}=2.6 \mathrm{~ms}, \mathrm{TE}=1.3 \mathrm{~ms}, \mathrm{FA}=35^{\circ}, \mathrm{FOV}=380 \times 356 \mathrm{~mm}^{2}$, voxel size $2 \times 2 \times 10 \mathrm{~mm}^{3}$ and SENSE factor $=2$. The MOLLI schema used was 5 beats (3s) 3 beats.

\section{T2 mapping}

Data for T2 mapping were acquired in three SAX planes (apical, mid-ventricular and basal). A navigator gated black blood prepared gradient spin-echo sequence (GraSE) was used [17] and 9 images were acquired. The imaging parameters for $1.5 \mathrm{~T}$ were: $\mathrm{TR}=1$ heartbeat, 9 echos, $\mathrm{TE} 1=12 \mathrm{~ms}, \Delta \mathrm{TE}=6.2 \mathrm{~ms}$, $\mathrm{FA}=90^{\circ}$, EPI factor: 3 , FOV $=380 \times 380 \mathrm{~mm}^{2}$, voxel size: $2 \times 2 \times 10 \mathrm{~mm}^{3}$ and SENSE factor $=2$. The imaging parameters for 3T were: $\mathrm{TR}=1$ heartbeat, 9 echos $\mathrm{TE} 1=11 \mathrm{~ms}, \Delta \mathrm{TE}=5.7 \mathrm{~ms}, \mathrm{FA}=90^{\circ}$, EPI factor: 3 , voxel size: $2 \times 2 \times 10 \mathrm{~mm}^{3}$ and SENSE factor $=2$.

\section{Image analysis}

\section{Evaluation of left and right ventricular function and mass}

Using Philips software (Extended Workspace, Philips Healthcare) the bSSFP cine images in SAX and $4 \mathrm{CH}$ were evaluated for wall motion abnormalities. The LV and RV ejection fraction were determined by manually contouring the endocardial borders in end-diastolic and end-systolic cardiac phase in SAX. The LV and RV mass, as well as the body surface indexed mass were determined by manually contouring of the endocardial and epicardial borders of both ventricles.

\section{T1 and T2 mapping - qualitative assessment}

All source images were assessed visually regarding artifacts caused by cardiac or respiratory motion or susceptibility. Artifacts were specified as focal, diffuse or band-shaped signal abnormalities of the myocardium (bright/white or dark/black) and led to exclusion of the affected segment. Some myocardial segments had to be excluded due to misplacement of the stack. Examples of artifacts that led to segment exclusions are illustrated in Fig. 1. The quality in consensus was analysed by two board-certified radiologists specializing in cardiac imaging.

\section{T1 and T2 mapping - quantitative assessment}

The QMASS 7.5-Software (QMASS 7.5 Enterprise solution with T1\&T2 mapping add-on, Medis Medical Imaging Systems, Leiden, Netherlands, www.
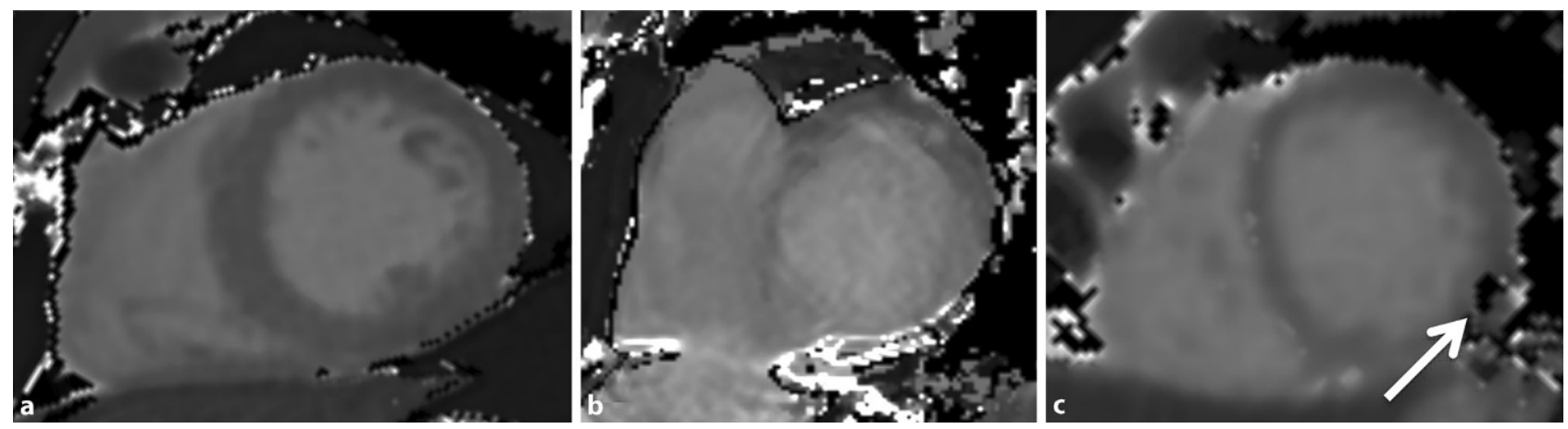

Fig. 1 Short axis view of the heart showing T1 mapping artifacts at 3T. Good quality map (a), motion artifact obscuring all segments in a slice which is too close to the base (b), susceptibility artifact in the inferolateral wall (white arrow) (c) 


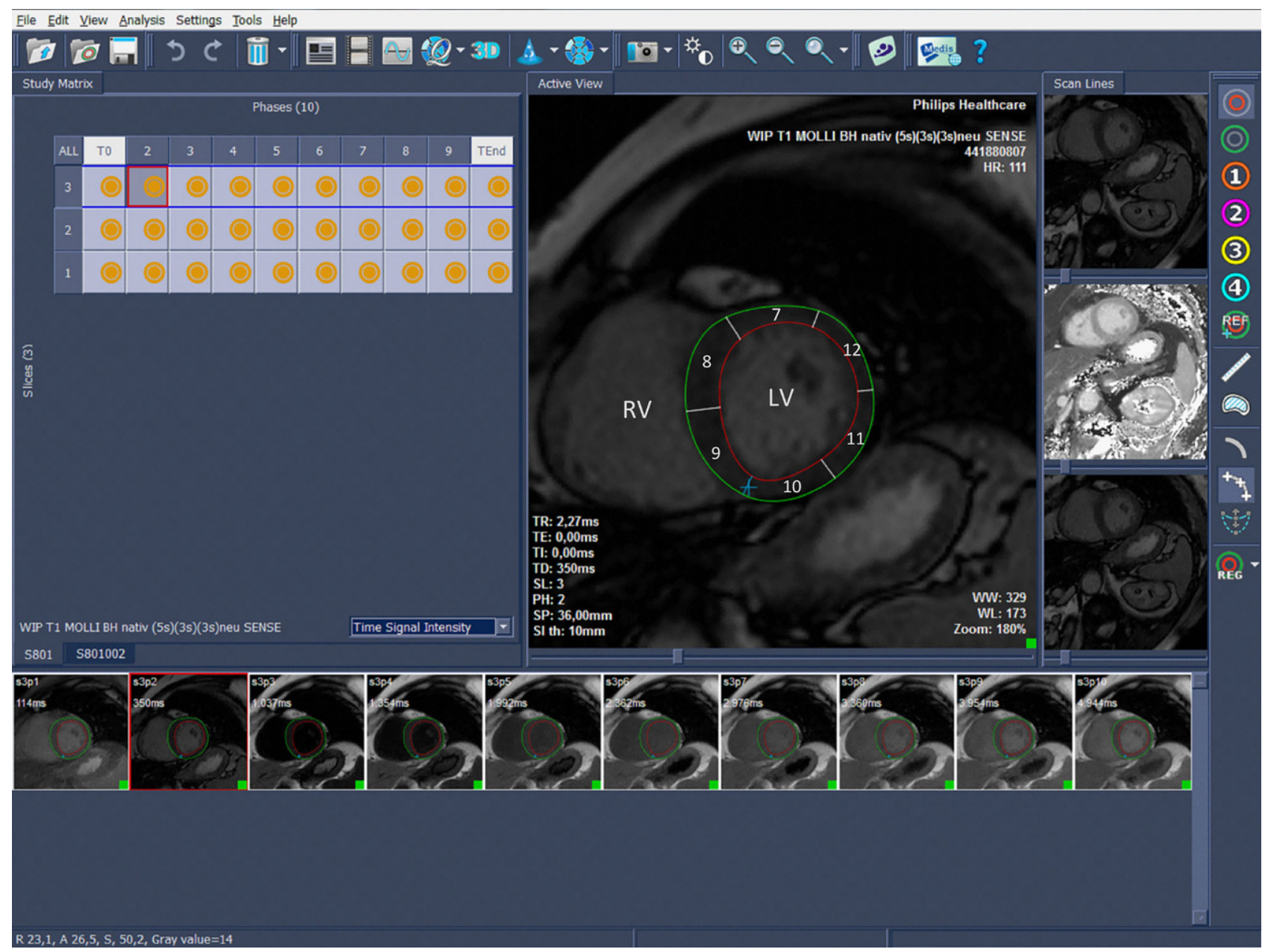

Fig. 2 Example of semiautomatic contouring and segmentation of the left ventricular myocardium in a mid-ventricular T1 short axis map. The table on the left shows the active slice (1-3) and number of the inversion time (T0- T9 and TEnd)

medis.nl) was used for generating the maps, segmentation and segmental quantification. The maps were generated by defining a region of interest (ROI) in the mid-ventricular septum. The endocardial and epicardial contours were manually drawn. To avert disturbances from the blood pool, $10 \%$ of the subendocardial and subepicardial aspect of the ROI were excluded automatically (Fig. 2). The myocardium was semiautomatically segmented into a 16 segment bull's-eye plot according to the American Heart Association (AHA-model, 6 basal segments, 6 mid-ventricular segments and 4 apical segments) [18]. Quantitative assessment was performed by two cardiac-imaging radiologist in consensus.

\section{Statistical methods}

Data were checked for consistency and normality. Means, standard deviations (SD), 95\% confidence intervals (CI), Pearson's correlation coefficient and which is displayed enlarged on the right side. On the right side segmentation according to the AHA 16-segment model is performed. Below, raw images ordered by inversion times are shown. $R V$ right ventricle, $L V$ left ventricle

1-factorial ANOVA model together with two-sided, independent t-tests were used to evaluate data. Normal ranges were estimated by using $2.5 \%$ and $97.5 \%$ percentiles to end up with $95 \%$ CI for the empirical distributions. Regression analysis was used to compare values between 1.5T and 3T. A $p$-value less than $5 \%$ indicates a statistical significance. All statistical analyses in this report were performed by use of STATISTICA 13 [19] and were done by one of the authors (WH). Data consistency was checked by analyzing the range of the variables. Data cleansing was done by validating and correcting values against a known list of entities and looking for unexpected or erroneous data entries. Normality was tested by using Kolmogorov-Smirnov tests.

\section{Results}

All 120 CMRI scans were carried out without adverse events. The scan was incomplete in one subject due to technical problems and two subjects had 
Fig. 3 Column graph illustrating the number of excluded segments due to artifacts depending on the individual mapping sequence and the heart segment

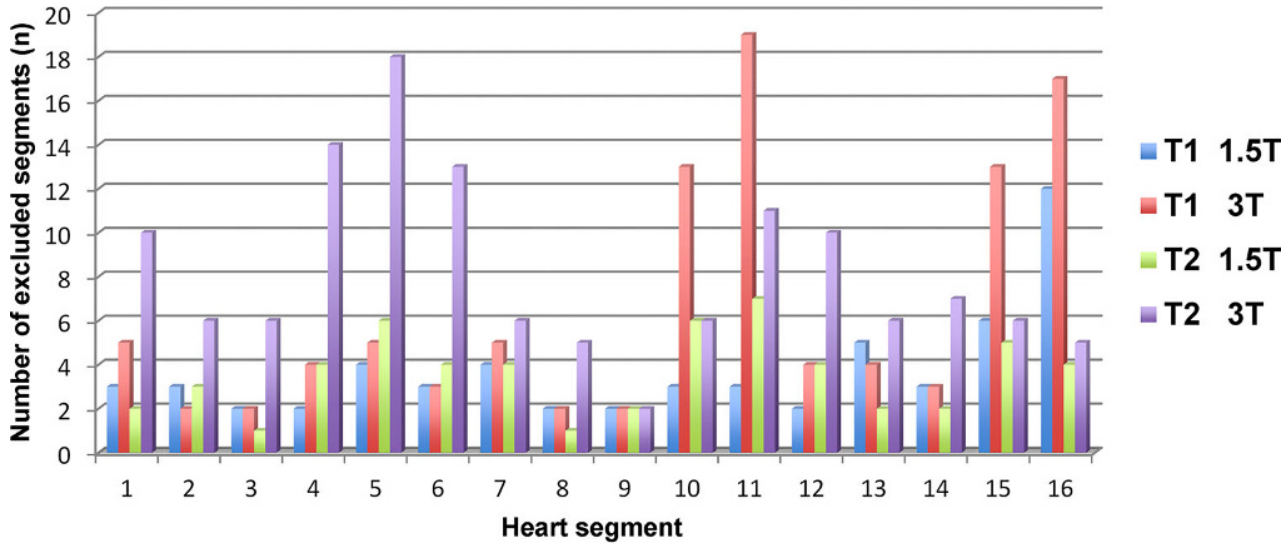

to be excluded due to the detection of a chronic disease (sarcoidosis and an atrial septal defect II, respectively). Therefore, 115 examinations in 58 subjects were available for further analysis. The $\mathrm{T} 1$ and T2 maps at $1.5 \mathrm{~T}$ were available for 57 subjects, T1 and $\mathrm{T} 2$ maps at $3 \mathrm{~T}$ were available for 58 subjects. Due to malpositioning of the basal slice too close to the atrioventricular plane 15 segments (1.6\%) had to be eliminated from the $\mathrm{T} 1$ measurements at $1.5 \mathrm{~T}, 10$ segments (1.1\%) from $\mathrm{T} 1$ at $3 \mathrm{~T}, 15$ segments $(1.6 \%)$ from T2 at $1.5 \mathrm{~T}$ and 21 segments $(2.3 \%)$ from T2 at 3T measurements. Females and males showed no statistically significant differences with respect to age, heart rate and body mass index (Table 1).

\section{T1 mapping}

At $1.5 \mathrm{~T}$ a total of 897 segments were available of which 59 segments were excluded due to artifacts (6.6\%). Particularly prone to artifacts was segment 16 (apicolateral wall). At 3T a total of 918 segments were available, of which 104 segments were excluded due to artifacts $(11.3 \%)$. The inferolateral wall in the apical and mid-ventricular slices (segments $10,11,15$ and 16) was especially prone to artifacts (Fig. 3).

Mean global relaxation times for both field strengths are presented in Table 2. We observed a prolongation of mean global $\mathrm{T} 1$ relaxation times at 3T compared to $1.5 \mathrm{~T}$ by $188 \mathrm{~ms}(1184 \pm 38 \mathrm{~ms}$ vs. $996 \pm 31 \mathrm{~ms})$.

Furthermore, the influences of basic volunteer characteristics on relaxation times were examined focusing on gender, age and since controversial data are presented in the literature, also on body mass index (BMI) and heart rate. No statistically significant differences in different age groups or in volunteers with a BMI above $25 \mathrm{~kg} / \mathrm{m}^{2}$ were found (Table 3).

Gender-specific and heart rate-specific global values are presented in Table 3. As previously discussed (Table 1), there were no significant differences regarding the basic characteristics between females and males; however, global T1 relaxation times were significantly longer in females (a detailed per segment delineation is presented in Fig. 4a). Furthermore, lower heart rates $(<70 \mathrm{bpm})$ were associated with significantly shorter global $\mathrm{T} 1$ relaxation times (Fig. 5a).

The next step was focused on slice and segmentspecific differences using a 16-segment model of the heart. Table 4 illustrates slice-dependent differences. Generally, T1 relaxation times (independent of field strength) were highest in the apical slice and lowest in the midventricular slice. Whereas $\mathrm{Tl}$ values at $1.5 \mathrm{~T}$ did not show significant slice-dependent differences $(p=0.057)$, at 3T significant differences were detectable between the base and the apical slice $(p=0.013)$ and furthermore between the midventricular and the apical slice $(p=0.002)$.

The T1 values reflect a high per segment correlation at $1.5 \mathrm{~T}$ and $3 \mathrm{~T}(\mathrm{r}=0.92, p<0.001)$ showing higher values in the septal and inferior segments and lower values in lateral segments (Fig. 6a).

In addition, since sex-specific significant differences were revealed, gender-specific bull's-eye plots were prepared. These are presented in Figs. 7 and 8 separately for females and males. Independently of gender, the lateral free wall showed lower T1 values than the septal region.

Table 2 Overall cohort, relaxation times in milliseconds for T1 and T2 at 1.5T and 3T

\begin{tabular}{|c|c|c|c|c|c|c|c|}
\hline & & Segments $(n)$ & Mean & Min. & Max. & $95 \% \mathrm{Cl}$ & SD \\
\hline \multirow[t]{2}{*}{$\mathrm{T} 1$} & $1.5 \mathrm{~T}$ & 838 & 995.8 & 916.8 & 1066.8 & $987.5-1004.2$ & 30.9 \\
\hline & $3 T$ & 814 & 1183.8 & 1118.9 & 1287.9 & $1173.8-1193.9$ & 37.5 \\
\hline \multirow[t]{2}{*}{ T2 } & $1.5 \mathrm{~T}$ & 840 & 55.8 & 46.3 & 62.1 & $55.0-56.5$ & 2.8 \\
\hline & $3 T$ & 776 & 51.6 & 44.5 & 61.4 & $50.8-52.4$ & 3.0 \\
\hline
\end{tabular}


Table 3 Differences in volunteer groups depending on gender, heart rate (HR), BMI (body mass index in $\mathrm{kg} / \mathrm{m}^{2}$ ) and age (years). Relaxation times in milliseconds (significant differences, $p<0.05$, italics)

\begin{tabular}{|c|c|c|c|c|c|c|}
\hline \multirow[t]{2}{*}{-} & & \multicolumn{2}{|c|}{ Female } & \multicolumn{2}{|l|}{ Male } & \multirow[t]{2}{*}{$p$} \\
\hline & & Mean & $S D$ & Mean & $S D$ & \\
\hline \multirow[t]{2}{*}{ T1 } & $1.5 T$ & 1004.6 & 31.5 & 984.4 & 26.5 & 0.015 \\
\hline & $3 T$ & 1196.2 & 38.8 & 1167.3 & 28.9 & 0.003 \\
\hline \multirow[t]{2}{*}{ T2 } & $1.5 T$ & 56.7 & 1.7 & 54.6 & 3.5 & 0.008 \\
\hline & $3 T$ & 52.0 & 3.0 & 51.0 & 2.9 & 0.2 \\
\hline \multirow[t]{2}{*}{-} & & \multicolumn{2}{|c|}{$H R<70$} & \multicolumn{2}{|c|}{$H R \geq 70$} & $p$ \\
\hline & & Mean & $S D$ & Mean & $S D$ & \\
\hline \multirow[t]{2}{*}{ T1 } & $1.5 T$ & 989.5 & 29.4 & 1011.1 & 29.7 & 0.017 \\
\hline & $3 T$ & 1176.8 & 33.3 & 1203.0 & 42.7 & 0.019 \\
\hline \multirow[t]{2}{*}{ T2 } & $1.5 T$ & 56.2 & 2.1 & 54.8 & 4.0 & 0.09 \\
\hline & $3 T$ & 52.1 & 2.9 & 50.1 & 2.9 & 0.02 \\
\hline \multirow[t]{2}{*}{ - } & & \multicolumn{2}{|c|}{$B M I \leq 25$} & \multicolumn{2}{|c|}{$\mathrm{BMI}>25$} & $p$ \\
\hline & & Mean & $S D$ & Mean & $S D$ & \\
\hline \multirow[t]{2}{*}{ T1 } & $1.5 T$ & 998.7 & 34.1 & 989.8 & 22.6 & 0.321 \\
\hline & $3 T$ & 1187.6 & 37.5 & 1175.8 & 37.3 & 0.278 \\
\hline \multirow[t]{2}{*}{ T2 } & $1.5 T$ & 55.9 & 2.8 & 55.6 & 3.0 & 0.731 \\
\hline & $3 T$ & 51.9 & 3.0 & 50.9 & 3.0 & 0.275 \\
\hline \multirow[t]{2}{*}{ - } & & \multicolumn{2}{|c|}{ Age $<45$} & \multicolumn{2}{|c|}{ Age $\geq 45$} & $p$ \\
\hline & & Mean & $S D$ & Mean & $S D$ & \\
\hline \multirow[t]{2}{*}{ T1 } & $1.5 T$ & 991.5 & 34.6 & 1000.9 & 25.5 & 0.264 \\
\hline & $3 T$ & 1184.1 & 41.9 & 1183.5 & 32.6 & 0.957 \\
\hline \multirow[t]{2}{*}{ T2 } & $1.5 T$ & 56.5 & 2.39 & 55.0 & 3.15 & 0.057 \\
\hline & $3 T$ & 51.3 & 2.31 & 51.9 & 3.63 & 0.442 \\
\hline
\end{tabular}

T1
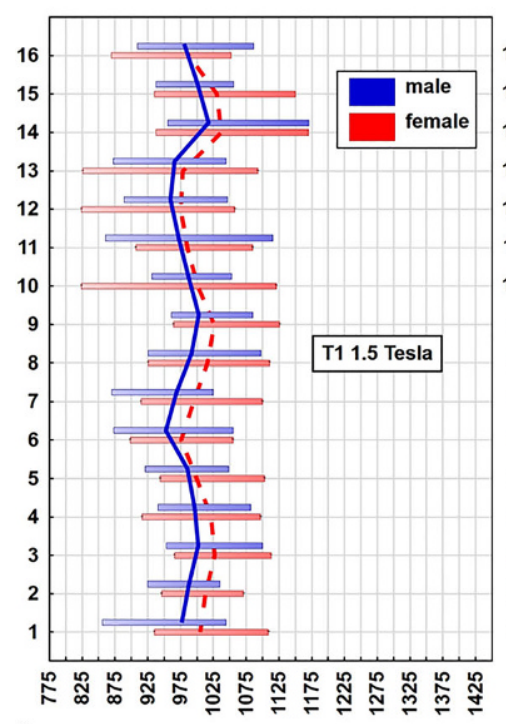

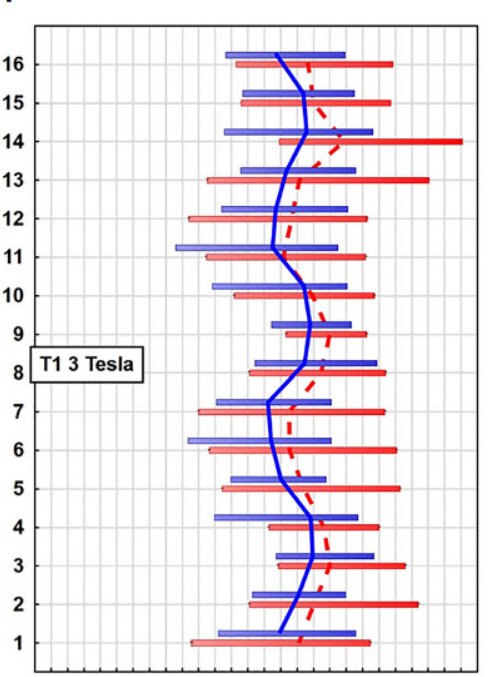

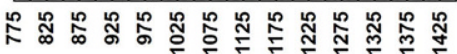

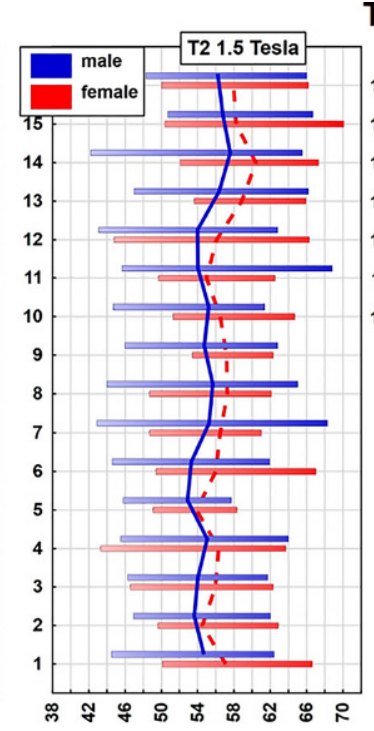

T2

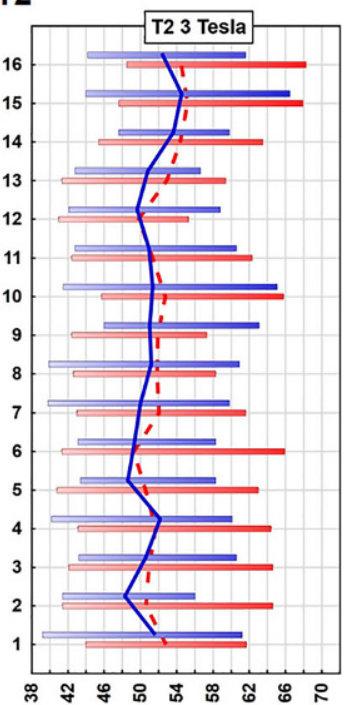

Fig. 4 Vertical plots illustrating per segment mean $\mathrm{T} 1$ (a) and $\mathrm{T} 2$ (b) relaxation times in milliseconds plus normal ranges $(95 \% \mathrm{Cl}$ for distributions): males = blue line, females = red dashed line, relaxation time in milliseconds on $\mathrm{x}$-axis, heart segment on $\mathrm{y}$-axis

Finally, the intra-individual correlation of $\mathrm{T} 1 \mathrm{re}-$ laxation times independent of field strength in each volunteer was investigated and a significant correlation of intra-individual global T1 values measured at $1.5 \mathrm{~T}$ and $3 \mathrm{~T}(\mathrm{r}=0.68$ and $p<0.001)$ could be confirmed (Fig. 9a).

\section{T2 mapping}

At $1.5 \mathrm{~T}$ a total of 897 segments were available, of which 57 were excluded due to artifacts $(6.4 \%)$. At $3 \mathrm{~T} 131$ segments out of 907 segments were excluded due to artifacts $(14.4 \%)$. The majority of excluded segments 
T1

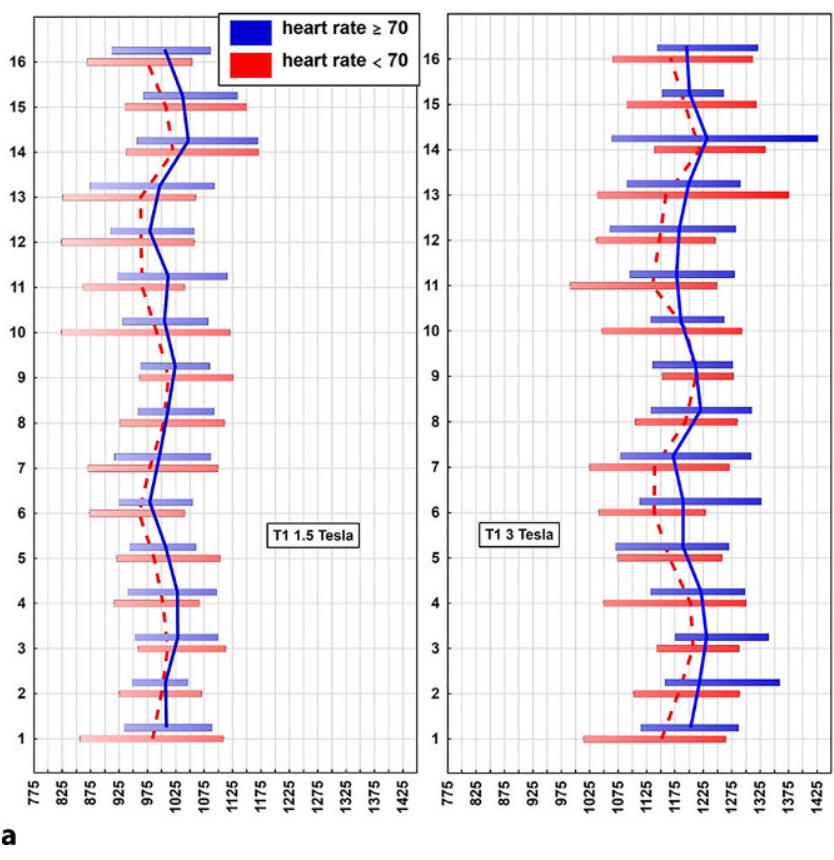

Fig. 5 Vertical plots illustrating per segment mean relaxation times in milliseconds plus normal ranges $(95 \% \mathrm{Cl}$ for distributions) for T1 (a) and T2 (b) at both field strengths. Heart

were in the basal slice, especially in the inferior and the lateral wall (Fig. 3). The same analysis was then performed as already described for T1 mapping. Mean global $\mathrm{T} 2$ relaxation times at $1.5 \mathrm{~T}$ and $3 \mathrm{~T}$ are presented in Table 2. According to published data [13, 20-22] $\mathrm{T} 2$ values were higher at $1.5 \mathrm{~T}$ as compared to $3 \mathrm{~T}$ (approximately $4.2 \mathrm{~ms}$ ). Similar to $\mathrm{T} 1 \mathrm{mapping}$, age and BMI did not influence T2 relaxation times (Table 3). Comparable to T1 mapping, females showed higher $\mathrm{T} 2$ values than males throughout all segments at 1.5T; however, at $3 \mathrm{~T}$ the prolongation of global $\mathrm{T} 2$ relaxation in females was not significant (Table 3; Fig. 4b).

Mean relaxation times of the cohort per slice are shown in Table 4. The T2 relaxation times for $1.5 \mathrm{~T}$ and
T2

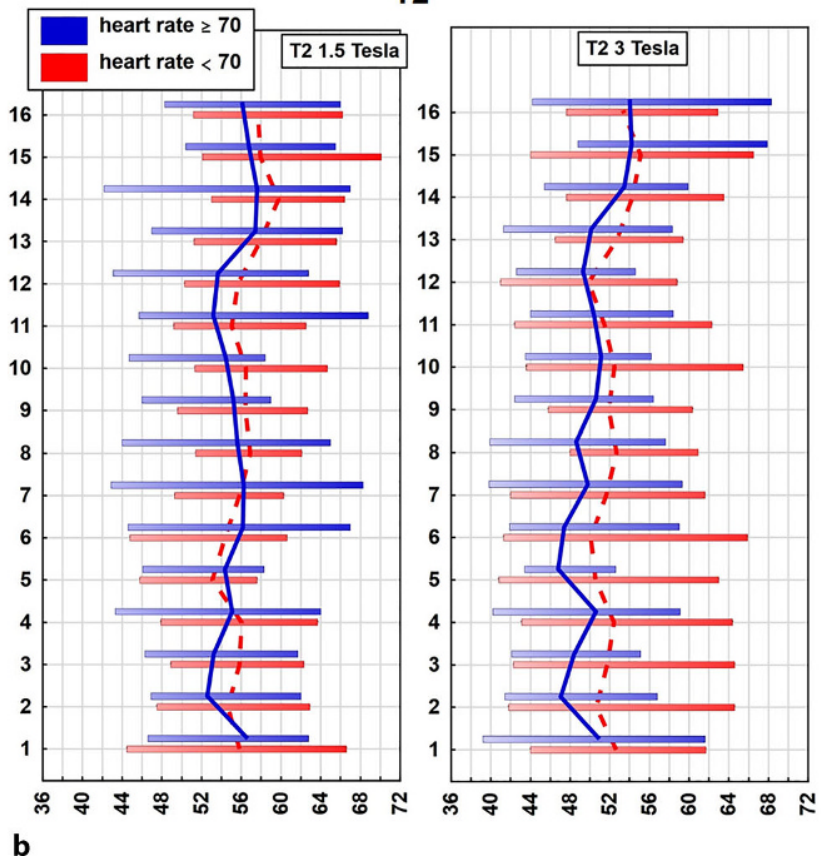

rate $\geq 70 \mathrm{bpm}=$ blue line, Heart rate $<70 \mathrm{bpm}=$ red dashed line, relaxation time in milliseconds on $\mathrm{x}$-axis, heart segment on y-axis

3T varied considerably depending on the slice, the values were highest in the apical slice and lowest in the basal slice. Significant differences of T2 values at $1.5 \mathrm{~T}$ between basal and middle slices $(p=0.019)$, basal and apical slices $(p<0.001)$ and moreover between middle and apical slices $(p<0.001)$ were present. Moreover, significant differences between T2 values at 3T of basal and apical slices $(p<0.001)$ and middle and apical slices $(p<0.001)$ were evident. Gender-specific values of T2 relaxation times are presented in a 16segment model of the heart (Figs. 7 and 8). Similar to $\mathrm{T} 1$ a significant per segment correlation of $\mathrm{T} 2 \mathrm{val}-$ ues was revealed $(r=0.82, p<0.001$ ) (Fig. 6b) showing highest values in the apical region.

Table 4 Per slice values, all volunteers, relaxation times in milliseconds

\begin{tabular}{|c|c|c|c|c|c|c|c|c|}
\hline & Position & $\begin{array}{l}\text { Valid seg- } \\
\text { ments }(n)\end{array}$ & Mean & Minimum & Maximum & $2.5 \%$ percentile & $\begin{array}{l}97.5 \% \text { per- } \\
\text { centile }\end{array}$ & SD \\
\hline \multirow[t]{3}{*}{$\mathrm{T} 11.5 \mathrm{~T}$} & Base & 310 & 997.0 & 931.7 & 1067.3 & 947.8 & 1057.5 & 31.3 \\
\hline & Middle & 326 & 991.1 & 900.8 & 1083.2 & 915.0 & 1074.3 & 33.6 \\
\hline & Apex & 202 & 1000.1 & 877.7 & 1098.3 & 919.5 & 1095.7 & 40.5 \\
\hline \multirow[t]{3}{*}{$\mathrm{T} 13 \mathrm{~T}$} & Base & 317 & 1182.3 & 1106.3 & 1313.7 & 1114.7 & 1273.0 & 40.9 \\
\hline & Middle & 302 & 1179.6 & 1100.8 & 1254.0 & 1111.0 & 1251.3 & 39.9 \\
\hline & Apex & 195 & 1194.4 & 1099.3 & 1322.0 & 1122.8 & 1299.3 & 46.1 \\
\hline \multirow[t]{3}{*}{ T2 1.5T } & Base & 307 & 54.8 & 47.3 & 60.7 & 47.6 & 59.9 & 3.0 \\
\hline & Middle & 318 & 55.5 & 43.5 & 62.5 & 44.9 & 61.5 & 3.3 \\
\hline & Apex & 215 & 57.8 & 47.0 & 65.7 & 49.3 & 64.8 & 3.6 \\
\hline \multirow[t]{3}{*}{ T2 3T } & Base & 260 & 50.4 & 42.8 & 63.0 & 43.1 & 59.2 & 4.4 \\
\hline & Middle & 308 & 51.1 & 42.4 & 60.0 & 43.2 & 59.7 & 3.3 \\
\hline & Apex & 208 & 53.6 & 46.9 & 61.6 & 47.2 & 61.0 & 3.0 \\
\hline
\end{tabular}


T1

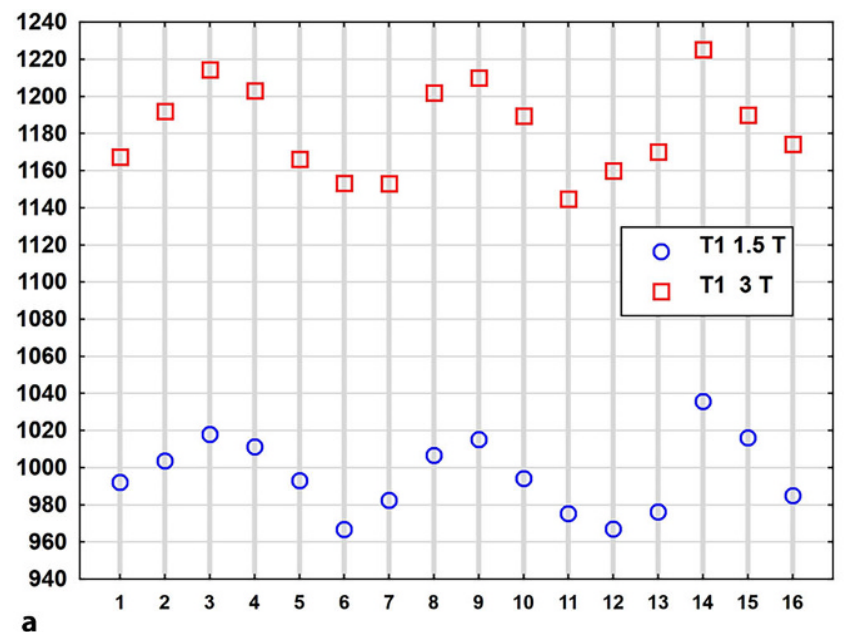

Fig. 6 Scatter plots, presenting per segment correlations of all available values for (a) T1 $(r=0.92, p<0.001)$ and for (b) T2 relaxation times in milliseconds $(r=0.82, p<0.001)$ at

Heart rate seemed to have an effect on T2 relaxation times. A significant reduction $(p=0.02)$ of global T2 relaxation times by $2.0 \mathrm{~ms}$ was revealed at $3 \mathrm{~T}$ in the subgroup of volunteers with a heart rate of $\geq 70 \mathrm{bpm}$. At $1.5 \mathrm{~T}$ the reduction of $\mathrm{T} 2$ relaxation times was not significant (Table 3). Mean values plus normal range for T2 relaxation times per segment of subjects with heart rates below $70 \mathrm{bpm}$ and with $70 \mathrm{bpm}$ and above are illustrated in Fig. 5b for 1.5T and 3T.

As described for $\mathrm{T} 1$ mapping a statistically significant correlation of intra-individual global T2 values measured at $1.5 \mathrm{~T}$ and $3 \mathrm{~T}$ after scanning each proband on both scanners was also found $(\mathrm{r}=0.40$ and $p=0.002$ ) (Fig. 9b).

\section{Discussion}

This study was designed to establish reference values for $\mathrm{T} 1$ and $\mathrm{T} 2$ relaxation times in healthy volunteers for our specific setting at 1.5T and 3T. Furthermore, the correlation between the intra-individual global T1 and T2 values at different field strengths was studied and published data concerning possible influences of physiological factors on relaxation times were reproduced.

As already pointed out in previous publications, different mapping techniques and field strengths are associated with a variety of normal values [20]. These values were comparable with publications using similar mapping protocols showing an overlap in confidence intervals; however, differences were also observed even in comparison with studies using MOLLI techniques for T1 and GraSE techniques for T2 mapping which were applied in this cohort ([20]; Table 2). As indicated by Kellman and Hansen [23] reasons for this inconsistency might be due to small variations in the study protocols (e.g. different flip angle, differ-
T2

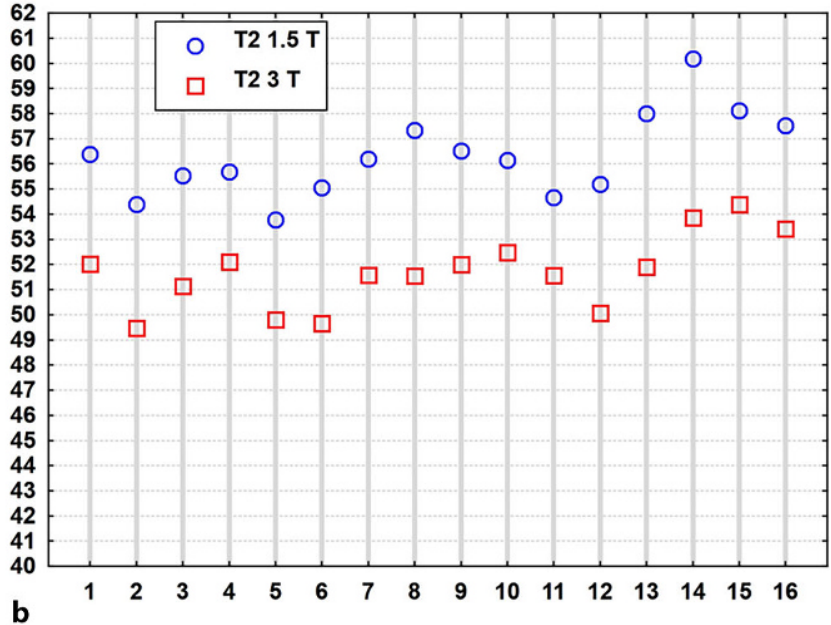

both field strengths. Heart segment on $\mathrm{x}$-axis, relaxation time in milliseconds on $y$-axis

ences in echo-spacing leading to partial volume errors, shimming, different MOLLI scheme). This has an important implication for clinical practice as apparently minor changes in mapping protocols might have an extensive impact on relaxation times. A recent study of Roy et al. [24] providing normal values using a 3T scanner from the same manufacturer but with a different MOLLI scheme with a 3(3)3(3)5 bSSFP sequence showed considerably lower $\mathrm{T} 1$ relaxation times of the whole heart than this study using a 5(3)3 bSSFP scheme $(1122 \pm 57 \mathrm{~ms}$ vs. $1184 \pm 38 \mathrm{~ms})$. This observation underlines that reference values have to be reassessed individually in every radiological department before implementing mapping techniques for routine CMRI examinations. The authors propose that a calibration of mapping protocols is necessary if myocardial $\mathrm{T} 1$ and $\mathrm{T} 2$ relaxation times are to be used as MRI biomarkers in clinical practice.

Apart from differences in study protocols patientrelated physiological factors are also presumed to affect relaxation times. Of note, various studies found an association between aging and lower $\mathrm{T} 1$ and higher T2 relaxation times $[17,21,25]$. In this institute more than two thirds of the CMRI clientele are below the age of 60 years. To avoid age-related alterations, this study focused on a younger population (only 4 subjects above the age of 60 years with a mean age of 41 years, ranging from 20 to 69 years). Consistently, in this cohort no significant differences of $\mathrm{T} 1$ and $\mathrm{T} 2 \mathrm{val}-$ ues between age groups ( $<45$ years of age vs. $\geq 45$ years of age) were found. Nevertheless, besides age gender and heart rate have also been discussed controversially as possible influencing factors for relaxation times $[13,21,25,26]$.

In this cohort, gender was a confounding factor for $\mathrm{T} 1$ and $\mathrm{T} 2$ relaxation times at $1.5 \mathrm{~T}$ and $3 \mathrm{~T}$. According to previous studies, females showed a significant pro- 

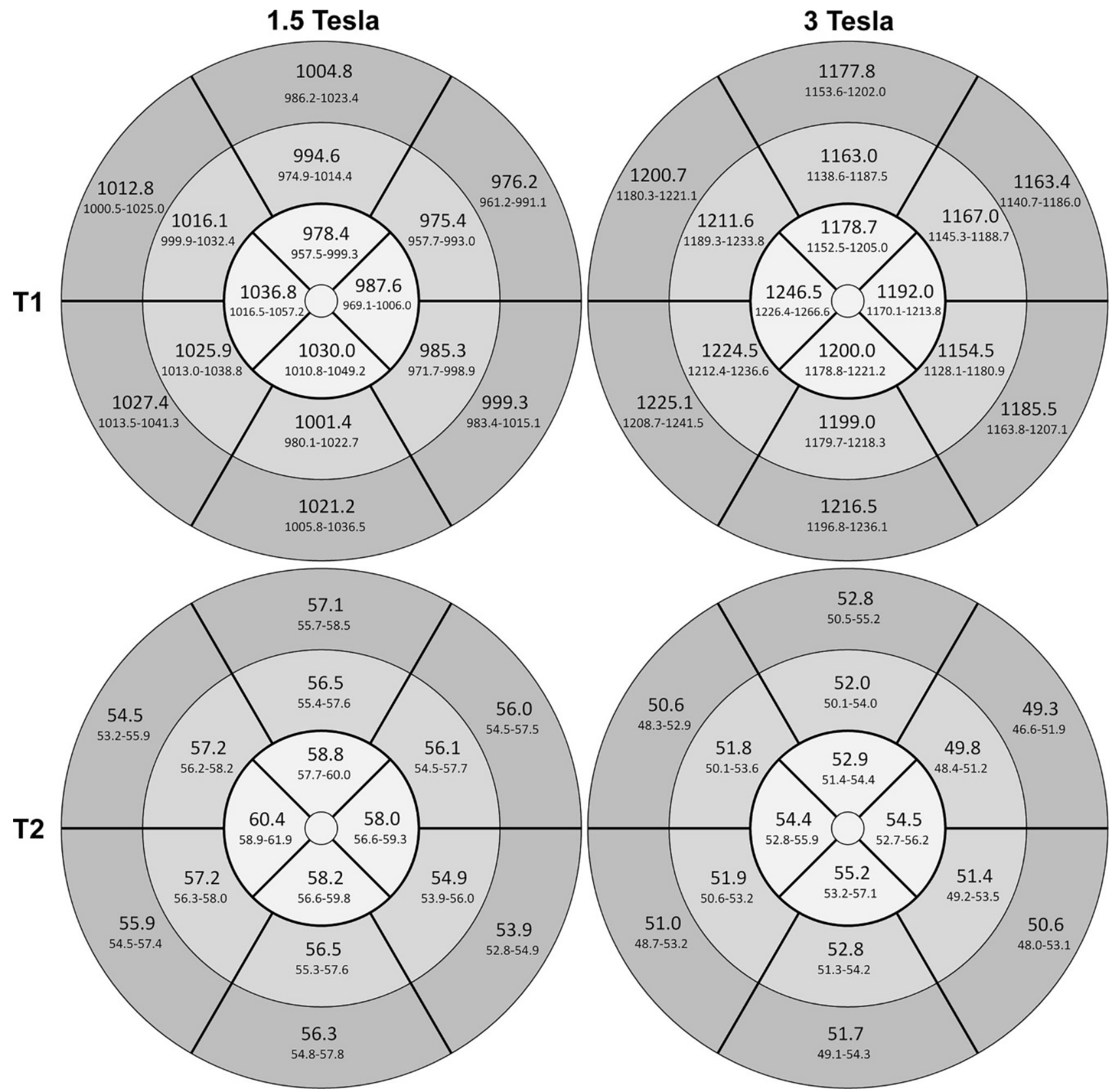

Fig. 7 16-segment heart model (AHA), bull's-eye plot of the left ventricle. Mean T1 and T2 relaxation times in milliseconds per segment plus $95 \% \mathrm{Cl}$ for females at $1.5 \mathrm{~T}$ (left) and at 3T (right)

longation of $\mathrm{T} 1$ relaxation times at $1.5 \mathrm{~T}$ and $3 \mathrm{~T}$ and of T2 relaxation times at $1.5 \mathrm{~T}[17,26-28]$; however, in accordance with von Knobelsdorff-Brenkenhoff et al. and Roy et al. [21, 24] no significant prolongation of $\mathrm{T} 2$ relaxation times in females at $3 \mathrm{~T}$ was observed which might be associated with numerous artifacts at $3 \mathrm{~T}$ in this study (Fig. 3). Furthermore, a heart rate $\geq 70 \mathrm{bpm}$ led to a significant global prolongation of T1 relaxation times at both field strengths. Even though the MOLLI 5(3)3 schema was used which is supposed to be less sensitive to higher heart rates plus a frequency adapted algorithm for higher heart rates, an effect seems to remain [29]. A possible explanation might be the influence of partial volume effects due to relatively thick slices $(8 \mathrm{~mm})$ and cardiac motion artifacts especially in thin myocardial walls [23].

The prolongation of $\mathrm{T} 1$ relaxation times in higher heart rates is in accordance with Piechnik et al. [26] while others described no significant influences [21]. In accordance with previous studies a heart rate of $\geq 70 \mathrm{bpm}$ was associated with a significant reduction of T2 relaxation times at 3T [21]. The small but significant influence of heart rate on $\mathrm{T} 1$ relaxation times and on $\mathrm{T} 2$ relaxation time at $3 \mathrm{~T}$ leads to the assumption that an algorithm for compensation of higher heart 

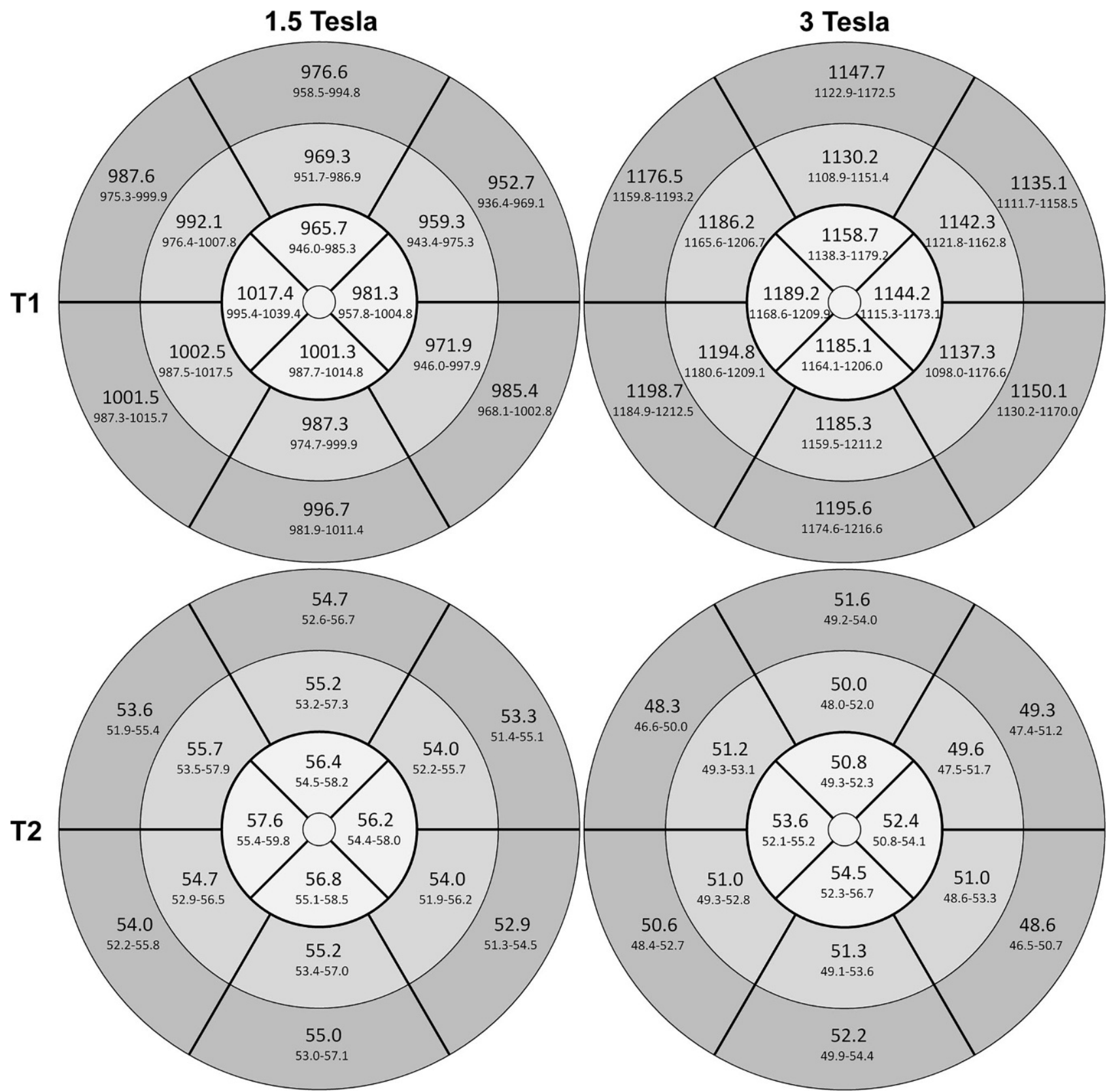

Fig. 8 16-segment heart model (AHA), bull's-eye plot of the left ventricle. Mean T1 and T2 relaxation time in milliseconds per segment plus $95 \% \mathrm{Cl}$ for males at $1.5 \mathrm{~T}$ (left) and at 3T (right)

rates is recommended to improve mapping quality for detection of subtle myocardial changes.

While a correlation of relaxation times at different field strengths in the same individual is presumed, to the best of our knowledge no study thus far has investigated this issue in a larger cohort. Therefore, we focused on this interesting subject and indeed were able to verify this association. This means that if a subject had low mean $\mathrm{T} 1$ values at 1.5T, these values were also low at 3T. As there are limited data on the reproducibility of myocardial $\mathrm{T} 1$ and $\mathrm{T} 2$ relaxation times at different field strengths in the same individual this is an important finding. The correlation was higher for T1 than for T2 values (Fig. 9). This might be associated with artifacts affecting T2 measurements especially at 3T, primarily off-resonance artifacts in regions adjacent to the lungs in addition to other artifacts, such as partial volume and motion artifacts. Of note, this is a critical point that becomes more relevant when evaluating the same individual at different field strengths. According to other publications, myocardial $\mathrm{T} 1$ relaxation times vary significantly with field strength. An increase of T1 values and a decrease of T2 values with higher field strength is reflected in previous studies $[20,28,30-34]$. The mean T2 value at 1.5T using a GraSE technique is consistent with other 


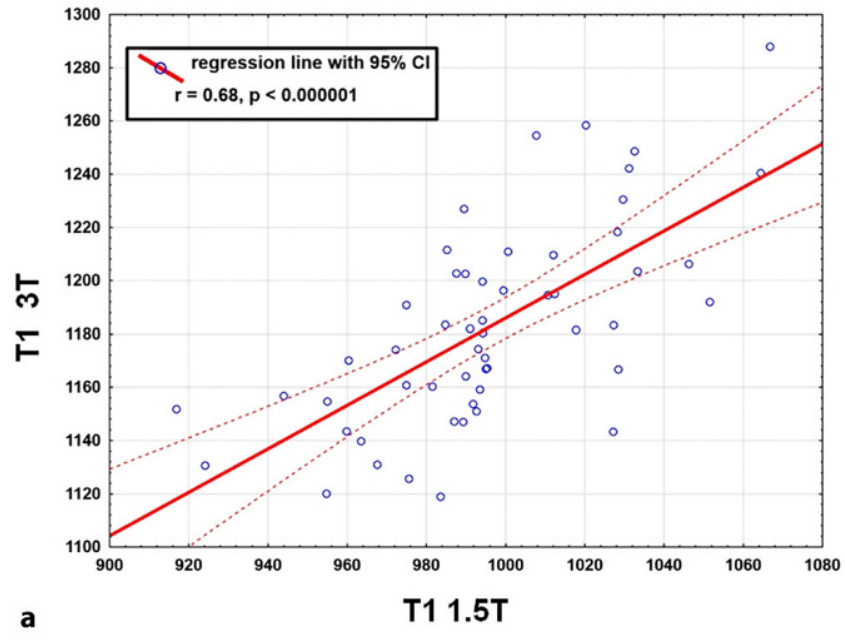

Fig. 9 Correlation of global T1 relaxation times in milliseconds for 1.5T and 3T (a) illustrates a good correlation $(r=0.68$, $p<0.000001$ ), T2 values (b) show a moderate but still signif-

publications [4, 13, 22, 24, 35]; however, while at 3T the T2 relaxation time was comparable with Baessler et al. using a GraSE sequence as applied in this study, other authors described considerably lower T2 values using accelerated multi-echo spin echo (MESE) or T2prepared bSSFP sequences [21, 36-38]. Again, this finding highlights the necessity of individual evaluation of normal values in every radiological institution. Furthermore, one can speculate that if institutional reference values are not available, only critical comparison with published relaxation times using similar protocols may be considered.

As already noted by other authors, also in zjis cohort the intrasubject and intersubject variability of $\mathrm{T} 1$ and especially of T2 values was considerably high per slice and per segment ([4, 21, 38], Table 4; Figs. 5, 6 and 7). This suggests a broad spectrum of normal values probably due to different effects of artifacts (e.g. B0, B1 inhomogeneities, off-resonance artifacts, motion artifacts, susceptibility artifacts or partial volume) in different areas of the myocardium. Apart from artifacts it has to be considered that e.g. regional differences in extracellular volume (ECV), amount of collagen, collagen fiber orientation and regional perfusion influence normal values. This insight complicates the distinction between borderline and pathologic relaxation times.

Due to respiratory or cardiac motion artifacts and susceptibility artifacts, a proportion of segments had to be excluded from this analysis (Fig. 3). Of note, the T2 maps at 3T emerged as the most vulnerable sequence. In contrast to Baessler et al. fewer visual artifacts were obtained using a comparable GraSE sequence at $1.5 \mathrm{~T}$ but exclusion rate at $3 \mathrm{~T}$ was almost the same $(6.4 \%$ vs. $20-24 \%$ at $1.5 \mathrm{~T}$ and $14.4 \%$ vs. $13-15 \%$ at 3T) [38]. The main cause of artifacts are B0 and B1 inhomogeneities which are especially problematic at $3 \mathrm{~T}$ leading to differences in the signal across im-

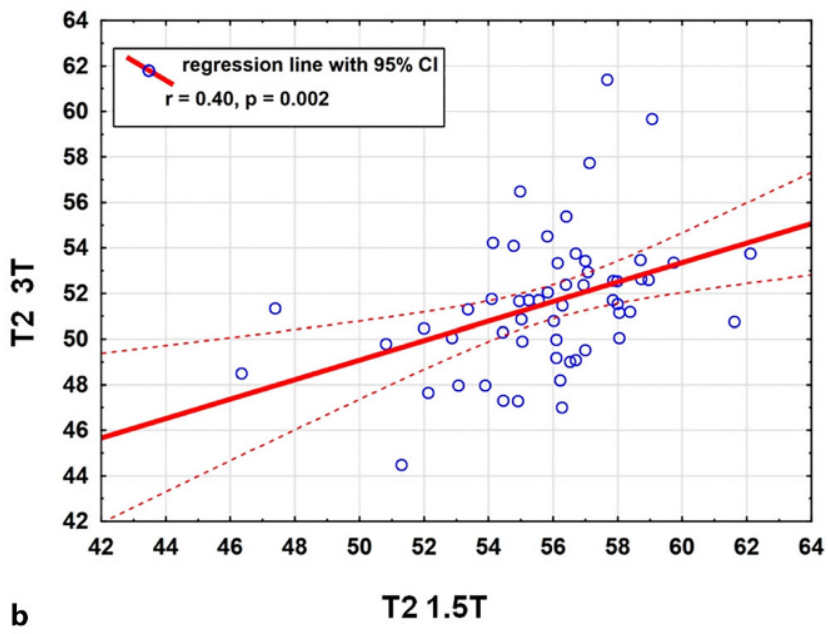

icant correlation $(r=0.4, p=0.002)$, dotted red lines indicate $95 \%$ confidence intervals for regression line

ages and between segmental measurements. Especially while using thick slice imaging partial volume effects cause artifacts particularly in apical slices and in hypermobile regions, such as the free lateral wall. Interestingly, as previously described [38], also in this study the elimination of segments affected by artifacts did not lead to exclusion of extreme value outliers.

In this study the topics of extracellular volume (ECV) and post-contrast T1-mapping were not elaborated, as it was pointed out in previous studies that native T1-mapping could differentiate between healthy and diseased myocardium with high sensitivity, specificity and diagnostic accuracy $[8,39]$. Even though noninvasive quantification of myocardial ECV via post-contrast T1-mapping is well validated [40, 41], native T1-mapping represents an excellent, rapid, noncontrast alternative for the detection of myocardial tissue remodelling [42]. Furthermore, The MOLLI 5(3)3 scheme used in this institution is known to have an excellent precision especially for native T1mapping but is not optimal for shorter $\mathrm{T} 1$ values associated with contrast [23].

There are several limitations to this study. This was a single center study of moderate sample size using MRI scanners from only one manufacturer. Therefore, a center-specific and manufacturer-specific bias cannot be excluded. No histological examination of the myocardium was performed. In the post-processing phase no program for motion correction was available which led to higher exclusion-rates of myocardial segments. Despite the relatively small size of the cohort results of studies using similar mapping techniques could be reproduced. This underlines the validity of these reference values and implications. As specified above, two thirds of the patients undergoing CMRI evaluation were below the age of 60 years. Therefore, this cohort consisted of younger volunteers, which might obscure a pre-described age dependency of re- 
laxation times in the aged myocardium. These normal values can only be applied and generalized in younger patients in institutions using similar mapping techniques to ours.

\section{Conclusion}

The results obtained during daily clinical practice in healthy volunteers indicated that $\mathrm{T} 1$ and $\mathrm{T} 2$ relaxation times showed gender and heart rate dependencies, even in T1 maps using a less heart rate sensitive MOLLI variant. Due to artifacts caution should be applied especially while performing $\mathrm{T} 2$ mapping at 3T and while measuring relaxation times in the inferolateral wall. As already pointed out in the consensus statement by the Society for Cardiovascular Magnetic Resonance (SCMR) endorsed by the European Association for Cardiovascular Imaging (EACVI) [14], we also highlight that reference values have to be reassessed in every radiological institution before implementing mapping protocols in daily clinical practice. Furthermore, to the best of our knowledge, the presumed correlation of intra-individual global $\mathrm{T} 1$ and $\mathrm{T} 2$ values measured at 1.5T and 3T could be demonstrated for the first time in a larger cohort.

Acknowledgements The authors thank Andrea Ladinger for support regarding organization and performing the MR examinations with her team, Bernhard Schnackenburg for the appropriation and optimization of mapping sequences and Kristen Kopp for English editing.

Funding Open access funding provided by Paracelsus Medical University.

Conflict of interest M. Granitz, L.J. Motloch, C. Granitz, M. Meissnitzer, W. Hitzl, K. Hergan, and A. Schlattau declare that they have no competing interests.

Open Access This article is distributed under the terms of the Creative Commons Attribution 4.0 International License (http://creativecommons.org/licenses/by/4.0/), which permits unrestricted use, distribution, and reproduction in any medium, provided you give appropriate credit to the original author(s) and the source, provide a link to the Creative Commons license, and indicate if changes were made.

\section{References}

1. Schulz-Menger J, Bluemke DA, Bremerich J, et al. Standardized image interpretation and post processing in cardiovascular magnetic resonance: Society for Cardiovascular Magnetic Resonance (SCMR) board of trustees task force on standardized post processing. J Cardiovasc Magn Reson. 2013;15:35.

2. Mewton N, Liu CY, Croisille P, Bluemke D, Lima JA. Assessment of myocardial fibrosis with cardiovascular magnetic resonance. JAm Coll Cardiol. 2011;57(8):891-903.

3. Messroghli DR, Niendorf T, Schulz-Menger J, Dietz R, Friedrich MG. T1 mapping in patients with acute myocardial infarction. J Cardiovasc Magn Reson. 2003;5(2):353-9.

4. Thavendiranathan P, Walls M, Giri S, et al. Improved detection of myocardial involvement in acute inflammatory cardiomyopathies using T2 mapping. Circ Cardiovasc Imaging. 2012;5(1):102-10.

5. Sado DM, White SK, Piechnik SK, et al. Identification and assessment of Anderson-Fabry disease by cardiovascular magnetic resonance noncontrast myocardial T1 mapping. Circ Cardiovasc Imaging. 2013;6(3):392-8.

6. Karamitsos TD, PiechnikSK, Banypersad SM, et al. Noncontrast T1 mapping for the diagnosis of cardiac amyloidosis. JACC Cardiovasc Imaging. 2013;6(4):488-97.

7. Sado DM, Maestrini V, Piechnik SK, et al. Noncontrast myocardial T1 mapping using cardiovascular magnetic resonance for iron overload. J Magn Reson Imaging. 2015;41(6):1505-11.

8. Puntmann VO, Carr-White G, Jabbour A, et al. T1-mapping and outcome in nonischemic cardiomyopathy: all-cause mortality and heart failure. JACC Cardiovasc Imaging. 2016;9(1):40-50.

9. Abdel-Aty H, Boye P, Zagrosek A, et al. Diagnostic performance of cardiovascular magnetic resonance in patients with suspected acute myocarditis: comparison of different approaches. JAm Coll Cardiol. 2005;45(11):1815-22.

10. Abdel-Aty H, Zagrosek A, Schulz-Menger J, et al. Delayed enhancement and T2-weighted cardiovascular magnetic resonance imaging differentiate acute from chronic myocardial infarction. Circulation. 2004;109(20):2411-6.

11. Oh-ID, RidgwayJP, KuehneT, etal. Cardiovascularmagnetic resonanceofmyocardial edemausing a shortinversion time inversion recovery (STIR) black-blood technique: diagnostic accuracy of visual and semi-quantitative assessment. J Cardiovasc Magn Reson. 2012;14:22.

12. Bohnen S, Radunski UK, Lund GK, et al. Tissue characterization by $\mathrm{T} 1$ and $\mathrm{T} 2$ mapping cardiovascular magnetic resonance imaging to monitor myocardial inflammation in healing myocarditis. Eur Heart J Cardiovasc Imaging. 2017;18(7):744-51.

13. Giri S, Chung YC, Merchant A, et al. T2 quantification for improved detection of myocardial edema. J Cardiovasc Magn Reson. 2009;11:56.

14. Messroghli DR, Moon JC, Ferreira VM, et al. Clinical recommendations for cardiovascular magnetic resonance mapping of T1, T2, T2* and extracellular volume: a consensus statement by the Society for Cardiovascular Magnetic Resonance (SCMR) endorsed by the European Association for Cardiovascular Imaging (EACVI). J Cardiovasc Magn Reson. 2017;19(1):75.

15. Burt JR, Zimmerman SL, Kamel IR, Halushka M, Bluemke DA. Myocardial T1 mapping: techniques and potential applications. Radiographics. 2014;34(2):377-95.

16. Messroghli DR, Greiser A, Frohlich M, Dietz R, SchulzMenger J. Optimization and validation of a fully-integrated pulse sequence for modified look-locker inversion-recovery (MOLLI) T1 mapping of the heart. J Magn Reson Imaging. 2007;26(4):1081-6.

17. Bonner F, Janzarik N, Jacoby C, et al. Myocardial T2 mapping reveals age- and sex-related differences in volunteers. JCardiovasc Magn Reson. 2015;17(1):9.

18. Schiller NB, Shah PM, Crawford M, et al. Recommendations for quantitation of the left ventricle by two-dimensional echocardiography. American Society of Echocardiography Committee on Standards, Subcommittee on Quantitation of Two-Dimensional Echocardiograms. J Am Soc Echocardiogr. 1989;2(5):358-67.

19. Hill T, Lewicki P. Statistics: methods and applications. A comprehensive reference for science, industry, and data mining. Tulsa: StatSoft; 2006.

20. Hamlin SA, Henry TS, Little BP, Lerakis S, Stillman AE. Mapping the future of cardiac MR imaging: case-based 
review of T1 and T2 mapping techniques. Radiographics. 2014;34(6):1594-611.

21. von Knobelsdorff-Brenkenhoff F, Prothmann M, Dieringer MA, et al. Myocardial T1 and T2 mapping at $3 \mathrm{~T}$ : reference values, influencing factors and implications. J Cardiovasc Magn Reson. 2013;15:53.

22. Wassmuth R, Prothmann M, Utz W, et al. Variability and homogeneity of cardiovascular magnetic resonance myocardial T2-mapping in volunteers compared to patients with edema. JCardiovasc Magn Reson. 2013;15:27.

23. Kellman P, Hansen MS. T1-mapping in the heart: accuracy and precision. J Cardiovasc Magn Reson. 2014;16:2.

24. Roy C, Slimani A, de Meester C, et al. Age and sex corrected normal reference values of $\mathrm{T} 1, \mathrm{~T} 2 \mathrm{~T} 2 *$ and $\mathrm{ECV}$ in healthy subjects at 3T CMR. J Cardiovasc Magn Reson. 2017;19(1):72.

25. Rauhalammi SM, Mangion K, Barrientos PH, et al. Native myocardial longitudinal (T1) relaxation time: regional, age, and sex associations in the healthy adult heart. J Magn Reson Imaging. 2016;44(3):541-8.

26. Piechnik SK, Ferreira VM, Lewandowski AJ, et al. Normal variation of magnetic resonance $\mathrm{T} 1$ relaxation times in the human population at $1.5 \mathrm{~T}$ using ShMOLLI. J Cardiovasc Magn Reson. 2013;15:13.

27. Liu CY, Liu YC, Wu C, et al. Evaluation of age-related interstitial myocardial fibrosis with cardiac magnetic resonance contrast-enhanced T1 mapping: MESA(Multi-EthnicStudy of Atherosclerosis). JAm Coll Cardiol. 2013;62(14):1280-7.

28. Carrick D, Haig C, Carberry J, et al. Microvascular resistance of the culprit coronary artery in acute ST-elevation myocardial infarction. JCI Insight. 2016;1(6):e85768.

29. Kellman P, Wilson JR, Xue H, Ugander M, Arai AE. Extracellular volume fraction mapping in the myocardium, part 1 : evaluation of an automated method. J Cardiovasc Magn Reson. 2012;14:63.

30. Kawel N, Nacif M, Zavodni A, et al. T1 mapping of the myocardium: intra-individual assessment of the effect of field strength, cardiac cycle and variation by myocardial region. J Cardiovasc Magn Reson. 2012;14:27.

31. Rogers T, Dabir D, Mahmoud I, et al. Standardization of T1 measurements with MOLLI in differentiation between health and disease - the ConSept study. J Cardiovasc Magn Reson. 2013;15:78.

32. Piechnik SK, Ferreira VM, Dall'Armellina E, et al. Shortened Modified Look-Locker Inversion recovery (ShMOLLI) for clinical myocardial T1-mapping at 1.5 and $3 \mathrm{~T}$ within a 9 heartbeat breathhold. J Cardiovasc Magn Reson. 2010;12:69.

33. Dabir D, Child N, Kalra A, et al. Reference values for healthy human myocardium using a T1 mapping methodology: results from the International T1 Multicenter Cardiovascular Magnetic Resonance Study. J Cardiovasc Magn Reson. 2014;16:69.

34. Dabir D, Rogers T, Voigt T, Schaeffter T, Nagel E, Puntmann VO.Age-gender referencevalues of native myocardial $\mathrm{T} 1$ at 1.5T and 3T: comparison of MOLLI and shMOLLI. JCardiovasc Magn Reson. 2013;15(1):1-2.

35. Bohnen S, Radunski UK, Lund GK, et al. Performance of $\mathrm{t} 1$ and $\mathrm{t} 2$ mapping cardiovascular magnetic resonance to detect active myocarditis in patients with recent-onset heart failure. Circ Cardiovasc Imaging. 2015; https://doi. org/10.1161/circimaging.114.003073.

36. Guo H, Au WY, Cheung JS, et al. Myocardial T2 quantitation in patients with iron overload at 3 T. J Magn Reson Imaging. 2009;30(2):394-400.

37. van Heeswijk RB, Feliciano H, Bongard C, et al. Freebreathing 3 T magnetic resonance T2-mapping of the heart. JACC Cardiovasc Imaging. 2012;5(12):1231-9.

38. Baessler B, Schaarschmidt F, Stehning C, Schnackenburg B, Maintz D, Bunck AC. A systematic evaluation of three different cardiac T2-mapping sequences at 1.5 and $3 \mathrm{~T}$ in healthyvolunteers. Eur J Radiol. 2015;84(11):2161-70.

39. Puntmann VO, Voigt T, Chen Z, et al. Native T1 mapping in differentiation of normal myocardium from diffuse disease in hypertrophic and dilated cardiomyopathy. JACC Cardiovasc Imaging. 2013;6(4):475-84.

40. Kammerlander AA, Marzluf BA, Zotter-Tufaro C, et al. T1 mapping by CMR imaging: from histological validation to clinical implication. JACC Cardiovasc Imaging. 2016;9(1):14-23.

41. Duca F, Kammerlander AA, Zotter-Tufaro C, et al. Interstitial fibrosis, functional status, and outcomes in heart failure with preserved ejection fraction: insights from a prospective cardiac magnetic resonance imaging study. Circ Cardiovasc Imaging. 2016; https://doi.org/10.1161/ circimaging.116.005277.

42. Shah RV, Kato S, Roujol S, et al. Native myocardial T1 as abiomarker of cardiac structurein non-ischemic cardiomyopathy. Am J Cardiol. 2016;117(2):282-8. 